\title{
Ionized gas, molecules and dust in Sh2-132
}

\author{
J. Vasquez, ${ }^{1,2 \star}$ C. E. Cappa, ${ }^{1,2}$ S. Pineault ${ }^{1,3}$ and N. U. Duronea ${ }^{1,2}$ \\ ${ }^{1}$ Instituto Argentino de Radioastronomia, CCT-La Plata, CONICET, C.C.5., 1894 Villa Elisa, Argentina \\ ${ }^{2}$ Facultad de Ciencias Astronómicas y Geofísicas, Universidad Nacional de La Plata, La Plata, Argentina \\ ${ }^{3}$ Départment de physique, de génie physique et d'optique and Centre de recherche en astrophysique du Québec (CRAQ), Université Laval, \\ Québec GIVOA6, Canada
}

Accepted 2010 February 24. Received 2010 February 10; in original form 2009 December 11

\begin{abstract}
We analyse the various interstellar components of the $\mathrm{H}$ II region Sh2-132. The main stellar source is the double binary system that includes the Wolf-Rayet star WR 153ab. We use radio continuum images at 408 and $1420 \mathrm{MHz}$, and $\mathrm{H}_{\mathrm{I}} 21-\mathrm{cm}$ line data taken from the Canadian Galactic Plane Survey, molecular observations of the ${ }^{12} \mathrm{CO}(1-0)$ line at $115 \mathrm{GHz}$ from the Five College Radio Astronomy Observatory, and available mid- and far-infrared observations obtained with the MSX and IRAS satellites, respectively.

Sh2-132 is composed of two shells showing radio continuum counterparts at both frequencies. The emission is thermal in nature. The estimated rms electron density and ionized mass of the nebula are $n_{\mathrm{e}} \simeq 20 \mathrm{~cm}^{-3}$ and $M_{\mathrm{H} \text { II }} \simeq 1500 \mathrm{M}_{\odot}$. The distribution of the CO emission shows molecular gas bordering the ionized nebula and interacting with it. The velocities of the molecular gas is in the range -38 to $-53 \mathrm{~km} \mathrm{~s}^{-1}$, similar to the velocity of the ionized gas.

The emission at $8.3 \mu \mathrm{m}$ reveals a ring-like feature of about $15 \mathrm{arcmin}$ that encircles the bright optical regions. This emission is due to the polycyclic aromatic hydrocarbons and marks the location of photodissociation regions.

The gas distribution in the environs of Sh2-132 can be explained in a scenario where the massive stars in the region photodissociated, ionized and swept up the dense molecular material from the parental cloud through their strong stellar winds and intense ultraviolet (UV) photon flux.
\end{abstract}

Key words: stars: individual: WR 153ab - stars: Wolf-Rayet - ISM: molecules - ISM: individual objects: Sh2-132 - ISM: bubbles - H II regions.

\section{INTRODUCTION}

The interaction between massive stars and the interstellar medium (ISM) leads to the formation of $\mathrm{H}$ in regions, interstellar bubbles and new stars. More specifically, the interaction between the molecular medium and the UV stellar radiation is at the origin of photodissociation regions (PDRs). Molecules are dissociated by UV photons in the range $6-13.6 \mathrm{eV}$. The gas component ranges from ionized atoms at the PDR surface to molecules deeper into the molecular region as the FUV flux is absorbed. The FUV flux illuminating these regions range within $1 \leq G_{0} \leq 10^{6}$, where $G_{0}$ is the intensity of the radiation field in the solar neighbourhood (Habing 1968), while the $\mathrm{H}_{\mathrm{I}}$ density lies within $10 \mathrm{~cm}^{-3} \leq n_{\mathrm{H}} \leq 10^{5} \mathrm{~cm}^{-3}$ (Tielens \& Hollenbach 1985; Sternberg \& Dalgarno 1989; Sternberg \& Dalgarno 1995; Bakes \& Tielens 1998). PDRs are formed at the edge of $\mathrm{H}$ II regions, planetary nebulae, reflection nebulae and stellar forming regions. Forbidden spectral lines in the far-infrared

\footnotetext{
^E-mail: jvasquez@ fcaglp.unlp.edu.ar; pete@iar-conicet.gov.ar
}

(FIR) as well as molecular lines are observed towards PDRs (e.g. Hogerheijde, Jansen \& van Dishoeck 1995; Kaufman et al. 1999).

An important component of PDRs is interstellar dust. Its presence plays a major role absorbing stellar radiation and re-emitting it in the mid-IR (MIR) and FIR. Polycyclic aromatic hydrocarbons (PAHs), which emit at 3.3, 6.2, 7.7, 8.6 and $11.3 \mu \mathrm{m}$, are tracers of PDRs (e.g. Watson et al. 2008).

In this paper, we study the interstellar environment of the $\mathrm{H}_{\mathrm{II}}$ region Sh2-132 (Sharpless 1959), located in the Perseus spiral arm, paying particular attention to the presence of PDRs at the interface between the ionized and molecular gas.

Sh2-132 was detected in $\mathrm{H} \alpha$, [O III] and [S II] lines by Heckathorn, Bruhweiler \& Gull (1982). The nebula is centred approximately at $(l, b) \simeq\left(102^{\circ} 50^{\prime},-0^{\circ} 42^{\prime}\right)$ and presents two bright regions, which are surrounded by diffuse emission regions. They can be identified in Fig. 1, which displays the Digitized Sky Survey (DSS)-R image of the nebula.

Churchwell \& Walmsley (1973) performed radio continuum observations at $2695 \mathrm{MHz}$ with an angular resolution of $18.2 \mathrm{arcmin}$, and, using measurements at several frequencies, derived a thermal 


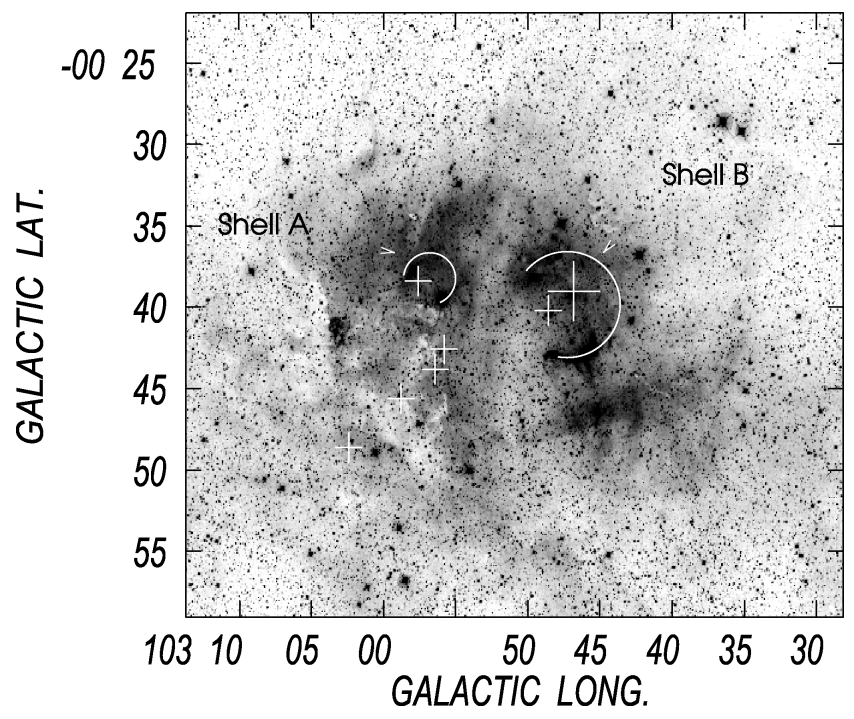

Figure 1. DSS-R image of the brightest section of Sh2-132. The grey-scale is arbitrary. The large cross marks the position of the WR star, while smaller crosses indicate the location of the OB stars.

spectral index. Harten, Felli \& Tofani (1978) investigated the characteristics and morphology of the nebula based on radio continuum observations at $610 \mathrm{MHz}$ using the Westerbork Synthesis Radio Telescope (WSRT) with a synthesized beam of 60 arcsec. Most of the emission at this frequency originates from two optically bright regions named Shell $\mathrm{A}$ and Shell $\mathrm{B}$, located at $(l, b) \simeq\left(102^{\circ} 57^{\prime}\right.$, $\left.-0^{\circ} 42^{\prime}\right)$ and $(l, b) \simeq\left(102^{\circ} 47^{\prime},-0^{\circ} 42^{\prime}\right)$. Both shells coincide with the bright optical regions within the nebula and are indicated in Fig. 1.

According to Harten et al. (1978), the optically bright section of Sh2-132 (at $l>102^{\circ} 43^{\prime}$ ) is an ordinary $\mathrm{H}_{\text {II }}$ region excited by the massive O-type star $\mathrm{BD}+55^{\circ} 2722$ and the WR star WR $153 \mathrm{ab}$ (= HD 211853), which are located close to the centre of the nebula and linked to Shell B. Chu, Treffers \& Kwitter (1983) reached to similar conclusions, based on the presence of the stars near the centre of the nebula and on the absence of velocity changes in the nebular spectra. The main parameters of these stars, as well as of other OB stars listed by Harten et al. (1978) that appear projected on to the $\mathrm{H}$ II region, are summarized in Table 1, which lists their Galactic coordinates, spectral classification, visual magnitude $V$, colour indices $(B-V)$ and $(B-V)_{0}$, visual absorption $A_{v}$, absolute magnitude $M_{V}$ and derived spectrophotometric distance $d$. Uncertainties in the derived spectrophotometric distances of some of the stars were estimated using $M_{V}$ values from Vacca, Garmany \& Shull (1996) and Landolt-Börnstein (1982). The large distance derived for $\mathrm{LS}$ III $+55^{\circ} 37$ casts doubts on their relation to the nebula. Note that, based on photometric data, Panov \& Seggewiss (1990) concluded that HD 211853 is a system with two pairs of stars, both hosting a WR component and an O-type star.

Chu \& Treffers (1981), based on Fabry-Perot observations of the $\mathrm{H} \alpha$ line, found that the ionized gas has LSR velocities in the range -53 to $-45 \mathrm{~km} \mathrm{~s}^{-1}$, in agreement with previous results from Georgelin \& Georgelin (1976, -50.9 $\mathrm{km} \mathrm{s}^{-1}$ ), Reynolds (1988, $\left.-48 \pm 1 \mathrm{~km} \mathrm{~s}^{-1}\right)$ and Fich et al. $\left(1990,-47.1 \mathrm{~km} \mathrm{~s}^{-1}\right)$. Quireza et al. (2006a) detected ionized gas at -50.46 and $-49.48 \mathrm{~km} \mathrm{~s}^{-1}$ from $\mathrm{He}$ and $\mathrm{H}$ radio recombination lines, respectively. Harten et al. (1978) estimated an emission measure $\mathrm{EM}=3000 \mathrm{pc} \mathrm{cm}^{-6}$ for the entire nebula.

New $\mathrm{H} \alpha$ and [O III] images taken by Miller \& Chu (1993) revealed an arc of 4 arcmin in size to the south of the WR star (at $\left.[l, b]=\left[102^{\circ} 46^{\prime},-0^{\circ} 43^{\prime}\right]\right)$. They concluded that the filament probably indicates the position of an ionization front and considered it to be a ring nebula related to the star. Based on optical data, Esteban \& Rosado (1995) found velocities in the range -41 to $-59 \mathrm{~km} \mathrm{~s}^{-1}$ for the arc and derived an electron density of $290 \pm 100 \mathrm{~cm}^{-3}$ from line ratios. The $\left[\mathrm{O}_{\mathrm{III}}\right] / \mathrm{H} \alpha$ ratio is consistent with values derived for ring nebulae, suggesting that WR $153 \mathrm{ab}$ is the main source of ionization (Esteban \& Rosado 1995). These authors classify the arc as $R_{\mathrm{s}}$ type based on the morphology of this feature and on the absence of a clear evidence of expanding motions in the line profiles. This last point suggests that the massive progenitor of the WR star contributed in the shaping of the nebula (see Chu 1981).

Distance estimates for the WR star based on different methods range from 2.75 to $5.0 \mathrm{kpc}$. Massey (1981) derived a spectrophotometric distance of $5.0 \mathrm{kpc}$, while van der Hucht (2001) estimated $2.75 \mathrm{kpc}$ based on its association to Cep OB1, with a distance uncertainty of 40 per cent. Foster \& Routledge (2003), using a method based on $\mathrm{H}$ i column densities, estimated $3.2 \pm 0.5 \mathrm{kpc}$. A distance of $3.68 \mathrm{kpc}$ was adopted by Churchwell \& Walmsley (1973), based on the WN6 + BOI spectral type of the WR star, known at that time. Georgelin \& Georgelin (1976) derived a distance of $3.6 \mathrm{kpc}$, while Fich, Blitz \& Stark (1989) obtained $4.2 \pm 1.5 \mathrm{kpc}$.

Adopting a mean LSR velocity of $-48 \mathrm{~km} \mathrm{~s}^{-1}$ for the ionized gas, and taking into account the presence of non-circular motions in this section of the Galaxy (see fig. 2a of Brand \& Blitz 1993), a kinematical distance $d_{\mathrm{k}}=3.5 \pm 1.0 \mathrm{kpc}$ can be predicted, in close agreement with distances derived optically for the WR star. We adopt this distance in this paper.

Table 1. OB-type stars towards the bright section of Sh2-132.

\begin{tabular}{|c|c|c|c|c|c|c|c|c|}
\hline Star & $(l, b)$ & ST & $\begin{array}{c}V \\
(\mathrm{mag})\end{array}$ & $\begin{array}{c}(B-V) \\
(\mathrm{mag})\end{array}$ & $\begin{array}{l}(B-V)_{0} \\
(\mathrm{mag})\end{array}$ & $\begin{array}{c}A_{v} \\
(\mathrm{mag})\end{array}$ & $\begin{array}{c}M_{V} \\
(\mathrm{mag})\end{array}$ & $\begin{array}{c}d \\
(\mathrm{kpc})\end{array}$ \\
\hline $\begin{array}{l}\text { HD } 211853 \\
\text { (WR 153ab) }\end{array}$ & $102^{\circ} 46^{\prime} .8,-0^{\circ} 39^{\prime}$ & $\mathrm{WN} 6 / \mathrm{WC}+\mathrm{O}^{a} \mathrm{I}^{a}$ & $9.08^{b}$ & $0.27^{b}$ & $b$ & $2.28^{b}$ & $-6.4^{b}$ & $2.75^{b}, 5.0^{c}$ \\
\hline LS III $+55^{\circ} 37$ & $102^{\circ} 46^{\prime} 8,-0^{\circ} 40^{\prime} .2$ & $\mathrm{O} 7 \mathrm{~V}^{d}$ & 11.01 & $0.32^{d}$ & $-0.29^{e}$ & 1.86 & $-5.2^{f}$ & 7.5 \\
\hline $\mathrm{BD}+55^{\circ} 2722$ & $102^{\circ} 48^{\prime} \cdot 6,-0^{\circ} 40^{\prime} \cdot 2$ & $\mathrm{O} 8.5 \mathrm{~V}^{d}$ & $9.91^{d}$ & $0.45^{d}$ & $-0.28^{e}$ & 2.26 & $-4.7^{f}$ & 3.0 \\
\hline $\mathrm{LS} \mathrm{III}+55^{\circ} 40$ & $102^{\circ} 56^{\prime} .4,-0^{\circ} 42^{\prime} \cdot 6$ & $\mathrm{O} 9 \mathrm{~V}^{d}$ & $12.08^{d}$ & $0.64^{d}$ & $-0.28^{e}$ & 2.85 & $-4.5^{f}$ & 5.6 \\
\hline $\mathrm{LS} \mathrm{III}+55^{\circ} 45$ & $102^{\circ} 59^{\prime} .4,-0^{\circ} 45^{\prime} \cdot 6$ & $\mathrm{O} 8^{d}$ & $10.45^{d}$ & $0.70^{d}$ & $-0.27^{f}$ & 3.01 & $-4.9,-5.8^{f, i}$ & $2.9-4.9$ \\
\hline LS III $+55^{\circ} 49$ & $103^{\circ} 02^{\prime} \cdot 4,-0^{\circ} 48^{\prime} \cdot 6$ & $\mathrm{~B}^{g}$ & 11.50 & 0.57 & $-0.26^{e}$ & 2.57 & $-2.45,-5.1^{f, h}$ & $1.9-6.4$ \\
\hline
\end{tabular}

${ }^{a}$ Smith, Shara \& Moffat (1998); ${ }^{b}$ van der Hucht (2001); ${ }^{c}$ Massey (1981); ${ }^{d}$ GOS Catalogue V2.2, Sota et al. (2008); ${ }^{e}$ Wegner (1994); ${ }^{f}$ Landolt-Börnstein (1996); ${ }^{g}$ SIMBAD data base; ${ }^{h}$ Spectral classification B0III-B2V was adopted; ${ }^{i}$ spectral class V and III vas adopted. 
Table 2. Observational parameters of the $\mathrm{H}_{\mathrm{I}}$ and ${ }^{12} \mathrm{CO}$ data.

\begin{tabular}{lcc}
\hline & H I & ${ }^{12} \mathrm{CO}(1-0)$ \\
\hline Original FWHM & $1.18 \times 0.98 \operatorname{arcmin}^{2}$ & $\sim 1$ arcmin \\
Final FWHM & $1.4 \times 1.4 \operatorname{arcmin}^{2}$ & \\
Velocity range (LSR) $\left(\mathrm{km} \mathrm{s}^{-1}\right)$ & $-165,+57$ & $-165,+57$ \\
Velocity resolution $\left(\mathrm{km} \mathrm{s}^{-1}\right)$ & 1.32 & 0.824 \\
rms noise $T_{\mathrm{b}}(\mathrm{K})$ & 1.7 & 0.15 \\
\hline
\end{tabular}

With the aim of investigating in some detail the nature of Sh2132, we analysed the distribution of the different gas components in its environs. To accomplish this goal we performed a multifrequency study using radio continuum data at several frequencies, $\mathrm{H}$ 21-cm line data, ${ }^{12} \mathrm{CO}(1-0)$ line data and IR observations at different wavelengths. These data revealed that PDRs are present at the interface between the ionized and molecular gas.

\section{DATA BASE}

Neutral hydrogen 21-cm line data from the Canadian Galactic Plane Survey (CGPS; Taylor et al. 2003) was used to analyse the H I gas distribution, while ${ }^{12} \mathrm{CO}(1-0)$ data at $115 \mathrm{GHz}$ from the Five College Radio Astronomy Observatory allowed us to study the molecular distribution. Intensities are shown as main beam brightness temperatures $T_{m b}$. The H I data were smoothed to $1.4 \times 1.4 \operatorname{arcmin}^{2}$ to facilitate the identification of the $\mathrm{H}_{\mathrm{I}}$ features. The main observational parameters of these data bases are detailed in Table 2.

Radio continuum observations at 408 and $1420 \mathrm{MHz}$ were also extracted from the CGPS. The image at $408 \mathrm{MHz}$ was obtained with a synthesized beam and an rms noise of $3.4 \times 2.8 \mathrm{arcmin}^{2}$ and $\sim 1.1 \mathrm{~K}$, respectively; the corresponding values for $1420 \mathrm{MHz}$ are $1.0 \times 0.81 \operatorname{arcmin}^{2}$ and $0.063 \mathrm{~K}$.

MIR images were extracted from the MSX Galactic Plane Survey (Price et al. 2001) at $8.3 \mu \mathrm{m}$ ( $A$ band), $12.1 \mu \mathrm{m}$ ( $C$ band), $14.7 \mu \mathrm{m}$ ( $D$ band) and $21.3 \mu \mathrm{m}$ ( $E$ band), with an angular resolution of 18.4 arcsec. MIR and FIR images at 12, 25, 60 and $100 \mu \mathrm{m}$ from the IRAS satellite (HIRES data) are also included. These images have angular resolutions in the range $0.5-2.0 \mathrm{arcmin}$.

\section{RESULTS}

\subsection{Radio continuum emission}

Figs 2 and 3 show the CGPS radio continuum emission at 1420 MHz. The grey-scale in Fig. 2 has been chosen to emphasize the large-scale region of faint emissivity to the north of WR 152 (Cappa et al. 2010, hence the 'burnt-out' appearance of the region around WR 153ab). The emission distribution at $1420 \mathrm{MHz}$ resembles an upside down ' $U$ '. This semicircular structure consists of two areas of strong emission coincident with Shells A and B detected by Harten et al. (1978), centred at $(l, b) \simeq\left(102^{\circ} 34^{\prime} 42^{\prime \prime} .4,-00^{\circ} 46^{\prime} 9^{\prime \prime} \cdot 8\right)$ and at $(l, b) \simeq\left(102^{\circ} 26^{\prime} 35^{\prime \prime} .8,-00^{\circ} 43^{\prime} 10^{\prime} .2\right)$, respectively. WR $153 \mathrm{ab}$, indicated by the larger cross in Fig. 1, appears projected on to the central region of Shell $\mathrm{B}$. Thus, the bright radio emission at $l \geq 102.5$ is in excellent agreement with the optical emission (see Fig. 1) and with the image at $610 \mathrm{MHz}$ by Harten et al. (1978). Both Shells A and B can be identified at $1420 \mathrm{MHz}$. Note in particular the small arc of emission at $(l, b)=(102.77,-0.73)$ (best identified in Fig. 3), which is the radio counterpart of the 4-arcmin arc detected at optical wavelengths by Miller \& Chu (1993).

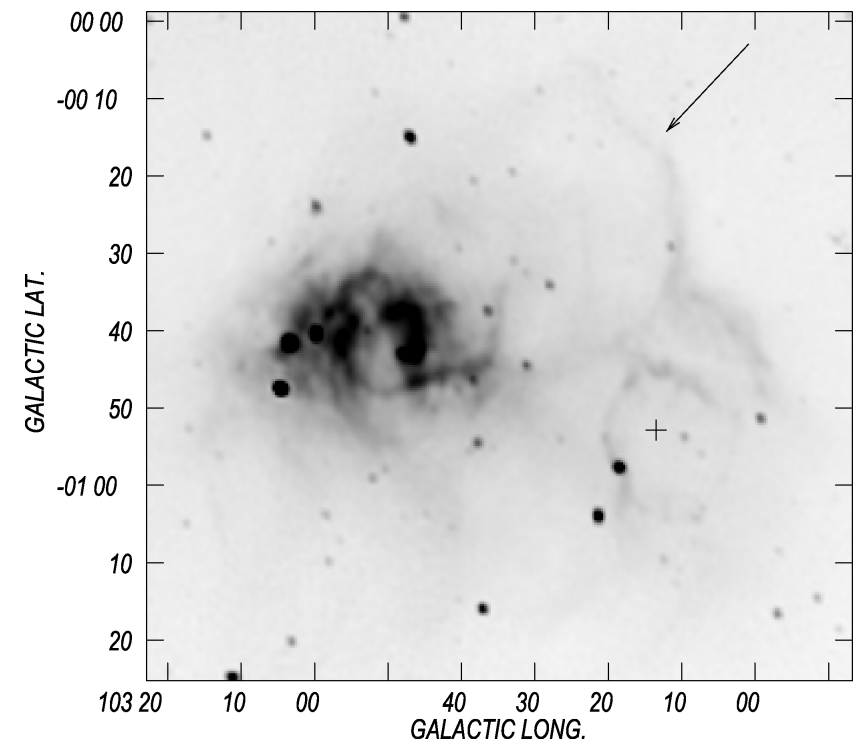

Figure 2. Full-resolution CGPS image at $1420 \mathrm{MHz}$ emphasizing faint emission to the north of WR 152 (indicated by a cross symbol in the lower right-hand part of the figure). The arrow points to a continuum feature corresponding to the large $\mathrm{H}$ i structure.

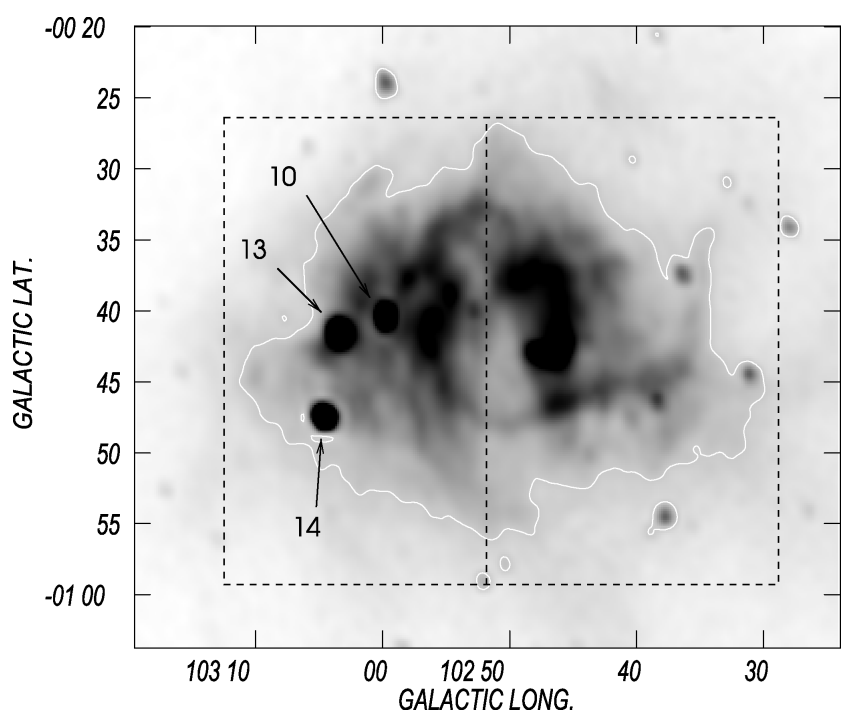

Figure 3. Full-resolution 1420-MHz CGPS image showing the eastern and western regions of Sh2-132 used for TT-plot analyses. The radio sources in direction to Sh2-132 are indicated by 10, 13 and 14. The white contour line marks the $9-\mathrm{K}$ contour of the convolved $1420-\mathrm{MHz}$ image used for the analysis. See text for details.

The faint structure centred at $(l, b)=(102.2,-0.83)$ is related to WR 152 and has been analysed in a previous paper (Cappa et al. 2010). Of particular interest is the curved filament indicated by an arrow in Fig. 2. This filament can be followed from $b \approx-0.75$ to nearly $b \approx-0.05$. The figure also shows a faint barely visible north-eastern section. This structure is likely the radio continuum counterpart of the $\sim 60$ arcmin diameter $\mathrm{H}_{\text {I }}$ structure discussed in Cappa et al. (2010).

Three bright point sources, best seen in Fig. 3, are projected on to the nebula near Shell A. Their coordinates, flux densities and identifications are included in Table 3 . The spectral index estimates indicate that sources 10 and 14 are non-thermal in nature and probably extragalactic, while source 13 is thermal. The value of the 
Table 3. Small diameter radio sources towards Sh2-132.

\begin{tabular}{|c|c|c|c|c|c|c|c|c|c|c|}
\hline$\#^{a}$ & $\begin{array}{l}l \\
b\end{array}$ & $\begin{array}{l}\mathrm{S}_{1420}{ }^{b} \\
(\mathrm{mJy})\end{array}$ & $\begin{array}{l}\mathrm{S}_{408} b \\
(\mathrm{mJy})\end{array}$ & $\alpha^{b}$ & $\begin{array}{l}\mathrm{S}_{178^{c}} \\
(\mathrm{mJy})\end{array}$ & $\begin{array}{l}\mathrm{S}_{365}{ }^{d} \\
(\mathrm{mJy})\end{array}$ & $\begin{array}{l}\mathrm{S}_{610}{ }^{e} \\
(\mathrm{mJy})\end{array}$ & $\begin{array}{l}\mathrm{S}_{1420}{ }^{f} \\
(\mathrm{mJy})\end{array}$ & $\mathrm{S}_{4850^{e}}$ & Identification \\
\hline 10 & $\begin{array}{l}102^{\circ} 59^{\prime} 50^{\prime \prime} 90 \\
-0^{\circ} 40^{\prime} 23^{\prime \prime} .35\end{array}$ & $195 \pm 10$ & 550 & -0.8 & & $819 \pm 87$ & 330 & & $<40$ & 7C $2218+558^{g}$ \\
\hline 13 & $\begin{array}{c}103^{\circ} 3^{\prime} 30^{\prime \prime} .24 \\
-0^{\circ} 41^{\prime} 28^{\prime \prime} .14\end{array}$ & $365 \pm 15$ & 280 & +0.2 & & & 160 & $222.6 \pm 7.5$ & $<40$ & NVSS J222034+561438 \\
\hline 14 & $\begin{array}{l}103^{\circ} 4^{\prime} 40^{\prime \prime} .04 \\
-0^{\circ} 47^{\prime} 27^{\prime \prime} .04\end{array}$ & $450 \pm 20$ & 1200 & -0.8 & $3100 \pm 500$ & $1738 \pm 62$ & 810 & & 60 & TXS 2219+559 \\
\hline
\end{tabular}

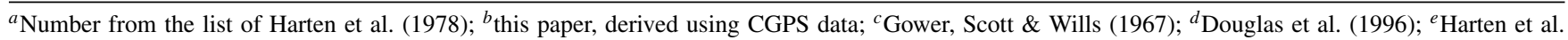
(1978); ${ }^{f}$ Condon et al. (1998); ${ }^{g}$ Waldram et al. (1996).

spectral index indicates that the radio source is optically thick at least at $408 \mathrm{MHz}$.

The CGPS image at $408 \mathrm{MHz}$ does not show as many details as the one at $1420 \mathrm{MHz}$, as it is of much lower resolution. However, it can be used to study the spectral index distribution of the radio continuum emission around WR 153ab. As a first step, we removed the point sources which are present over the region of interest and convolved the 1420 - and $408-\mathrm{MHz}$ images to the same spatial resolution of 3.4 arcmin. We then constructed a TT plot, in which the brightness temperature $T_{\mathrm{b}}$ at one frequency is plotted point by point against the brightness temperature at the other frequency. The brightness temperature spectral index $\beta$, where $T_{\mathrm{b}} \propto v^{\beta}$, is directly related to the slope of the straight line fitted by regression, $\beta=-\log ($ slope $) / \log (1420 / 408)$. The usual flux-density spectral index $\alpha\left(S_{v} \propto v^{\alpha}\right)$ is simply $\alpha=\beta+2$. The TT-plot method is immune from errors in the zero level of the individual images (which the simpler technique of dividing one map by the other, even after background subtraction, is not).

Three different TT plots were constructed, one for the entire Sh2-132 region and one for the eastern and western halves, which correspond to the two shells identified by Harten et al. (1978). Fig. 3, which is the full-resolution image at $1420 \mathrm{MHz}$, shows these different regions, the dashed lines delimiting the eastern and western parts. The three bright small diameter sources coincide with sources 10, 13 and 14 in table 2 of Harten et al. (1978) and had been subtracted from the original image before convolution to a circular beam of 3.4 arcmin.

The results of the TT-plot analysis are presented in Fig. 4. In each case, the analysis was limited to regions brighter than $9 \mathrm{~K}$ at $1420 \mathrm{MHz}$ and $80 \mathrm{~K}$ at $408 \mathrm{MHz}$. Although a significant amount of scatter is present in the diagram, especially for the eastern sector, there is no evidence for the presence of significant variations of the spectral index and a further study of spatial variations is not warranted. The best-fitting spectral index is $\alpha=-0.03 \pm 0.03$, corresponding to thermal emission.

In order to obtain some radio continuum parameters, we first derive the total number $N_{\mathrm{u}}$ of UV ionizing photons per second required to produce the observed free-free emission. This can be written as (e.g. Chaisson 1976)

$N_{\mathrm{u}}=0.76 \times 10^{47} T_{4}^{-0.45} v_{\mathrm{GHz}}^{0.1} S_{v} d_{\mathrm{kpc}}^{2}$,

where $S_{v}$ is the flux density in Jy, $T_{4}$ is the electron temperature in units of $10^{4} \mathrm{~K}$ and $v_{\mathrm{GHz}}$ is the frequency in $\mathrm{GHz}$. We use the CGPS image at $1420 \mathrm{MHz}$. The derived flux density is significantly dependent on how the source dimension is defined. The surrounding background is somewhat variable, nevertheless it is reasonable to assume that the source emission corresponds to brightness temperatures above $6.5-7 \mathrm{~K}$. Using an average background of $7 \mathrm{~K}$, we obtain a flux density at $1420 \mathrm{MHz}$ of $23 \pm 5 \mathrm{Jy}$, the error being determined from a second estimate using a background of $6.5 \mathrm{~K}$. From the above equation for $N_{\mathrm{u}}$ and taking $T_{4}=0.8$ (Quireza et al. 2006b), we obtain $N_{\mathrm{u}}=2.2 \times 10^{49} \mathrm{~s}^{-1}$. Considering two O6I-type stars and two WN6 as components of the multiple system, and the stars BD $+55^{\circ} 2722$, LS III $+55^{\circ} 45$ and LS $+55^{\circ} 40$ (see Table 1 ), we obtain the UV ionizing photons per second emitted by the stars, $Q_{0}=(16.6 \pm 3.0) \times 10^{49} \mathrm{~s}^{-1}$, assuming solar metallicity (Smith, Norris \& Crowther 2002).

The comparison between the number of UV photons emitted by the stars $Q_{0}$ and the number of UV photons used to ionize the gas $N_{\mathrm{u}}$ indicates that the massive stars in the nebula are responsible for the ionization. A large fraction of the UV photons are also used to heat the associated interstellar dust. This result differs from the one by Harten et al. (1978) since their estimate of $Q_{0}$ was too low.

We can now use the models of Mezger \& Henderson (1967) to infer the properties of the extended ionized gas, namely its mass $M_{\mathrm{HII}}$ and the rms electron number density $n_{\mathrm{e}}$. Letting $\theta_{\mathrm{Ga}}$ be the observed half-power width of the extended emission (in minutes of arc, after correction for the beam), we obtain

$n_{\mathrm{e}}\left(\mathrm{cm}^{-3}\right)=6.35 \times 10^{2} u_{1} a^{-1 / 2} T_{4}^{0.175} v_{\mathrm{GHz}}^{0.05} S_{v}^{0.5} \theta_{\mathrm{Ga}}^{-1.5} d_{\mathrm{kpc}}^{-0.5}$,

$M_{\mathrm{H} \text { II }}\left(\mathrm{M}_{\odot}\right)=0.386 u_{2} a^{-1 / 2} T_{4}^{0.175} v_{\mathrm{GHz}}^{0.05} S_{v}^{0.5} \theta_{\mathrm{Ga}}^{1.5} d_{\mathrm{kpc}}^{2.5}$,

where $a\left(v, T_{\mathrm{e}}\right)$ is the Gaunt factor (about unity for our purposes), $d_{\mathrm{kpc}}$ is the distance in kpc and the parameters $u_{1}$ and $u_{2}$, both of order unity, depend on the assumed model for the source. These parameters are given by Mezger \& Henderson (1967) for three different idealized models. From Fig. 3, it is apparent that the nebulosity can be roughly broken up into two different regions separated by a nearly vertical line running at a longitude of $\sim 102.87$. These two regions correspond to shells A and B from Harten et al. (1978). The flux densities of the two subregions are $11 \pm 2$ and $12 \pm 3 \mathrm{Jy}$, respectively. For simplicity, we model each half-region as a sphere of constant density and of angular size $12 \mathrm{arcmin}$. From Mezger \& Henderson (1967), we find $u_{1}=0.78, u_{2}=1.29$. Assuming $T_{4}=$ 0.8 , from which we determine $a^{-1 / 2}=1.04$, we finally have an rms $n_{\mathrm{e}}=20 \mathrm{~cm}^{-3}$ and $M_{\mathrm{H} \text { II }}=1500 \mathrm{M}_{\odot}$ for each region, where, given the error in estimating fluxes, we have simply taken $S_{v}=10 \mathrm{Jy}$ for each half. The derived rms electron density is compatible with estimates by Harten et al. (1978) for Shells A and B.

\subsection{IRAS and MSX emission}

The IRAS $(60 \mu \mathrm{m})$ and $M S X$ band $A(8.3 \mu \mathrm{m})$ emissions are shown in Fig. 5. Although we analysed the four IRAS bands, we include the $60 \mu \mathrm{m}$ image only since the extended emission is clearly seen at this wavelength. Emission at $8.3 \mu \mathrm{m}$ (upper panel) in diffuse 

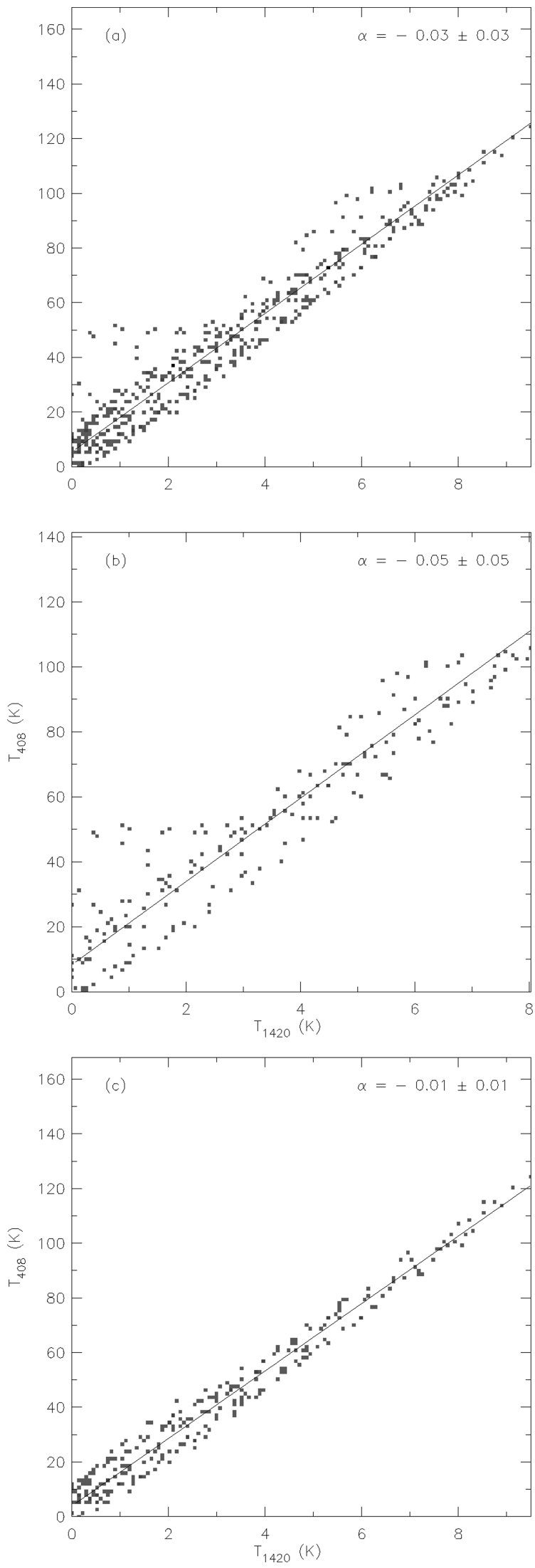

Figure 4. TT plots corresponding to (a) the whole structure, (b) the eastern half and (c) the western half. For all plots, a background of 9 and $80 \mathrm{~K}$ has been subtracted from the convolved images at 1420 and $408 \mathrm{MHz}$, respectively.
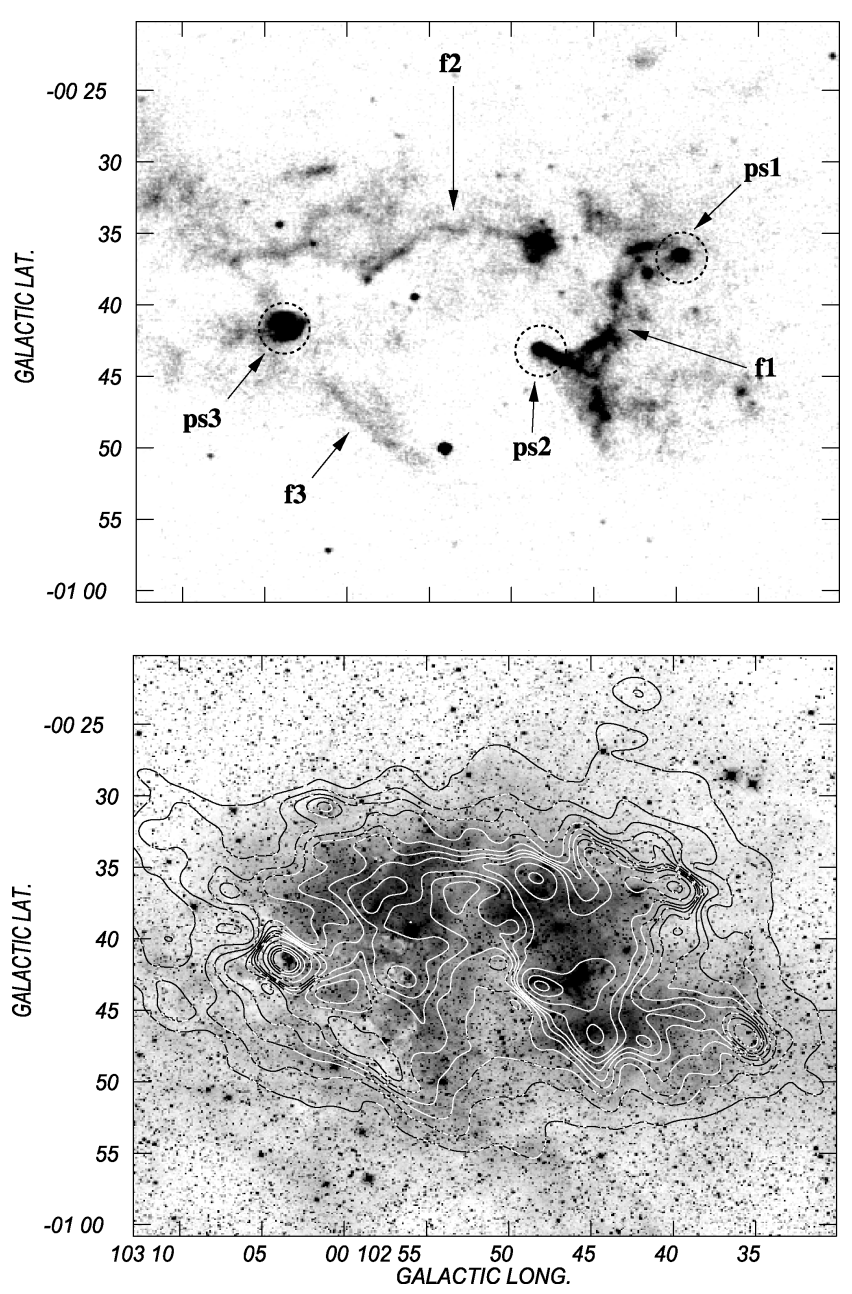

Figure 5. Upper panel: Sh2-132 at $8.3 \mu \mathrm{m}$ (MSX band $A)$. The grey-scale goes from $8.0 \times 10^{-7}$ to $3.5 \times 10^{-6} \mathrm{~W} \mathrm{~m}^{-2} \mathrm{sr}^{-1}$. Extended filaments and point-like sources are indicated (see text). Bottom panel: overlay of the DSS$\mathrm{R}$ image and the image at $60 \mu \mathrm{m}$. IR contours are 50-190 $\mathrm{MJy} \mathrm{sr}^{-1}$ in steps of $20 \mathrm{MJy} \mathrm{sr}^{-1}$, and from 300 to $900 \mathrm{MJy} \mathrm{sr}^{-1}$ in steps of $200 \mathrm{MJy} \mathrm{sr}^{-1}$.

nebulae has a contribution from both PAH and dust continuum. As pointed out by Povich et al. (2007), in very energetic regions like M 17, dust continuum can be very strong. However, for the case of Sh2-132, the assumption that MSX band $A$ is dominated by PAH emission seems to be reasonable. Consequently, emission in this band indicates the presence of PDRs, while the emission in the FIR originates in large dust grains.

The image at $8.3 \mu \mathrm{m}$ (upper panel) reveals the existence of a ringlike feature centred at $(l, b)=\left(102^{\circ} 53^{\prime},-00^{\circ} 40^{\prime}\right)$ of about $\sim 15^{\prime}$ in size. This feature can be also identified at $60 \mu \mathrm{m}$ (lower panel). The comparison of the optical emission with the emission at $8.3 \mu \mathrm{m}$ shows that the MIR emission encircles most of the nebula, following the outer rim of the ionized gas. This fact is more noticeable for Shell B and is particularly striking near $(l, b)=\left(102^{\circ} 47^{\prime},-00^{\circ} 43^{\prime}\right)$, where the 4 -arcmin ring nebula is present. The bottom panel shows that the emission distribution at $60 \mu \mathrm{m}$ resembles that in the optical.

In order to estimate some dust parameters, we have divided this ring-like structure in three main filaments, named f1, f2 and f3, which are indicated in the upper panel of Fig. 5. Note that $\mathrm{f} 3$ borders the region with high extinction seen in the optical image near $(l, b)=$ $\left(103^{\circ} 0^{\prime},-00^{\circ} 47^{\prime}\right)$. 
Table 4. IR filaments and point sources.

\begin{tabular}{|c|c|c|c|c|c|c|c|c|c|c|}
\hline \multirow[t]{2}{*}{ Source } & \multicolumn{8}{|c|}{ Fluxes (Jy) } & \multirow[t]{2}{*}{$T_{\mathrm{d}}(\mathrm{K})$} & \multirow[t]{2}{*}{$L_{I R A S}\left(L_{\odot}\right)$} \\
\hline & $8.3 \mu \mathrm{m}$ & $12.1 \mu \mathrm{m}$ & $14.7 \mu \mathrm{m}$ & $21.3 \mu \mathrm{m}$ & $12 \mu \mathrm{m}$ & $25 \mu \mathrm{m}$ & $60 \mu \mathrm{m}$ & $100 \mu \mathrm{m}$ & & \\
\hline f1 & $46 \pm 1$ & - & - & - & $72 \pm 4$ & $192 \pm 24$ & $1370 \pm 130$ & $2765 \pm 145$ & $32 \pm 7$ & 43800 \\
\hline f3 & $1.8 \pm 0.3$ & - & - & - & $4.9 \pm 0.5$ & $32 \pm 11$ & $133 \pm 40$ & $343 \pm 37$ & $32 \pm 6$ & 5000 \\
\hline ps1 & 1.45 & 1.85 & 0.97 & 2.14 & 3.03 & 4.28 & 74.1 & 274 & $28 \pm 5$ & 2700 \\
\hline ps2 & 2.22 & 3.93 & 3.78 & 7.02 & 7.8 & 16.1 & 106 & 318 & $30 \pm 5$ & 3900 \\
\hline
\end{tabular}

A number of interesting point-like sources located on to the filaments can also be identified in the MSX and IRAS images. They are indicated in the figure as ps1, ps2 and ps3. IR source ps1 at $(l, b)=\left(102^{\circ} 39^{\prime} 45^{\prime \prime},-00^{\circ} 36^{\prime} 24^{\prime \prime}\right)$ is the MSX source G102.662700.6067 , coincident with IRAS $22160+5551$; ps2 at $(l, b)=$ $\left(102^{\circ} 48^{\prime} 18^{\prime \prime},-00^{\circ} 43^{\prime} 6^{\prime \prime}\right)$ corresponds to G102.8051-00.7184 and IRAS $22172+5549$; while ps 3 at $(l, b)=\left(103^{\circ} 03^{\prime} 44^{\prime \prime},-00^{\circ} 41^{\prime} 27^{\prime \prime}\right)$ is G103.0624-00.6911 and IRAS 22187+5559.

After background subtraction, the IR flux densities of the filaments in the four IRAS bands and in the MSX band $A$ were calculated. These values are listed in Table 4 . The quoted errors reflect the uncertainties in the determination of the background. Emission in bands $C, D$ and $E$ is very weak in comparison with the strong background emission leading to large uncertainties in flux-density estimates. Values for these bands are not included. The three point sources, whose flux densities in the MSX and IRAS bands are included in the table, can be classified as young stellar object (YSO) candidates following criteria by Junkes, Fuerst \& Reich (1992) and Lumsden et al. (2002) for IRAS and MSX point sources, respectively. In the case of the IRAS criteria, Junkes et al. (1992) consider (i) $S_{100}>20 \mathrm{Jy}$, (ii) $1.2<S_{100} / S_{60}<6$, (iii) $S_{60} / S_{25} \geq 1$ and (iv) $Q_{60}+Q_{100} \geq 4$, where $S_{i}$ is the flux density in the bands centred at 25,60 and $100 \mu \mathrm{m}$, and $Q_{i}$ is the quality of the fluxes at these bands. The criteria of Lumsden et al. (2002) are (i) $S_{21} / S_{8}>2$ and (ii) $S_{14} / S_{12}>1$, where $S_{i}$ is the flux density centred at $8.3,12.1$, 14.7 and $21.3 \mu \mathrm{m}$.

It has been demonstrated (Scoville \& Kwan 1976) that the IR spectrum of radiatively excited dust grains can be fitted by a modified Planck function $B\left(T_{\mathrm{d}}\right) v^{m}$, where $v^{m}$ is the grain emissivity and $m$ increases from 1 to 2 depending on the wavelength and composition of dust grains (Schwartz 1982). Adopting $m=1.5$, according with the molecular environment (Whittet 1994), and using the measured flux densities at 60 and $100 \mu \mathrm{m}$, we derived the dust colour temperature $T_{\mathrm{d}}$ summarized in Table 4 . IR luminosities for the point sources are also included. IR filaments are excited by the intense stellar radiation field of WR 153ab and the other massive stars in the region. The slightly higher dust colour temperature derived for f1 can be explained bearing in mind that this filament is closer to the exciting stars than $\mathrm{f} 2$ and f3. In Fig. 6, the MIR and FIR spectral energy distributions corresponding to the filaments are shown. Note that the measured fluxes at shorter wavelengths exceed the values of our model, possibly as the result of the emission of nebular lines which may contribute to the IR flux densities.

\subsection{Molecular gas distribution}

The analysis of the ${ }^{12} \mathrm{CO}(1-0)$ emission distribution leads to the detection of two structures with velocities in the ranges from -53.4 to $46.0 \mathrm{~km} \mathrm{~s}^{-1}$, and from -46.0 to $-38.6 \mathrm{~km} \mathrm{~s}^{-1}$. Fig. 7 shows the

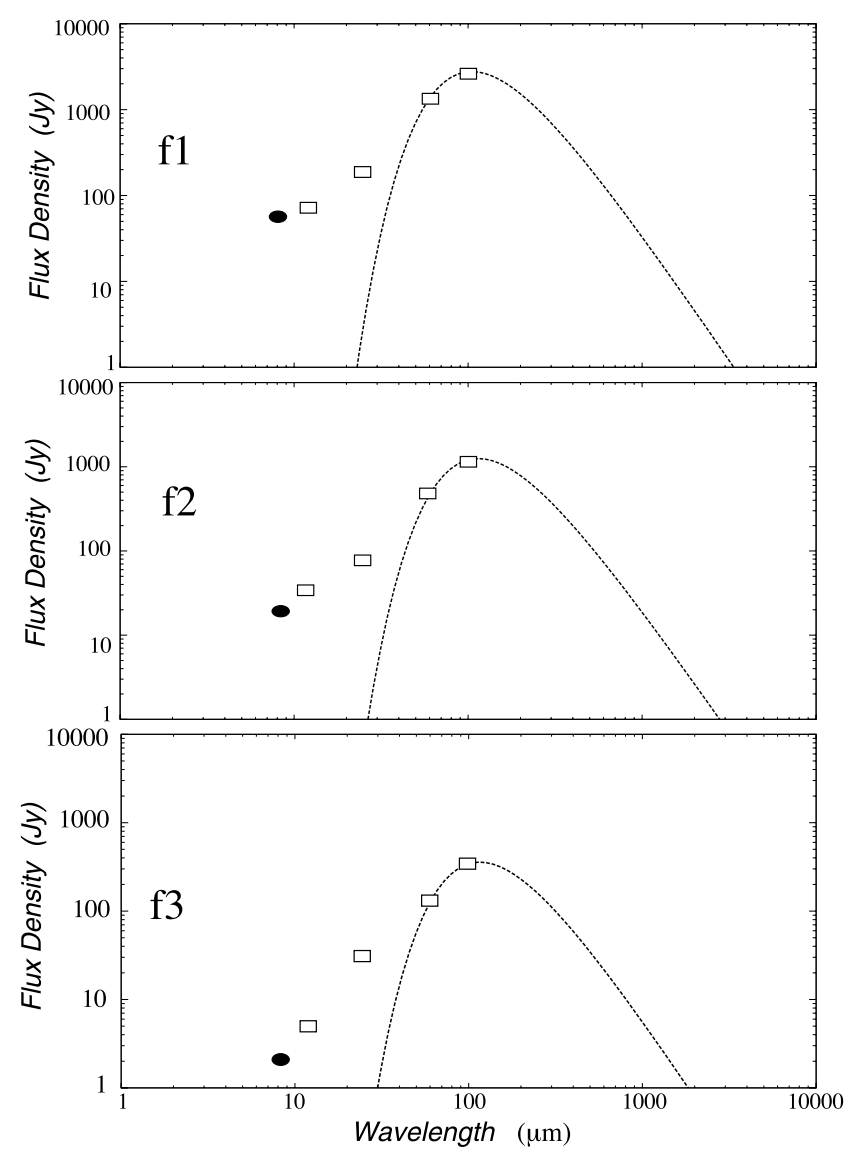

Figure 6. IR spectral energy distribution for the IR filaments. The lines in the diagram corresponding to $\mathrm{f} 1, \mathrm{f} 2$ and $\mathrm{f} 3$ show the emission of a blackbody with a $T=32,29$ and $32 \mathrm{~K}$, respectively, and a $v^{1.5}$ emissivity law. The boxes denote flux densities in the four IRAS bands, and the circle corresponding to the $M S X$ band $A$.

averaged $\mathrm{CO}$ emission (contours) for the two molecular structures superposed on to the emission at $8.3 \mu \mathrm{m}$.

The CO feature present between -46.0 and $-38.6 \mathrm{~km} \mathrm{~s}^{-1}$ (upper panel) exhibits a ring-like appearance between $l \sim 102^{\circ} 35^{\prime}$ and $102^{\circ} 50^{\prime}$, while for $l>102^{\circ} 45^{\prime}$, the emission is concentrated along $b \simeq-0^{\circ} 35^{\prime}$. The whole structure will be referred to as cloud A. Two smaller molecular clouds are detected at $(l, b) \sim\left(103^{\circ} 2^{\prime}\right.$, $-0^{\circ} 30^{\prime}$ ) (indicated in the figure as cloud C) and at $(l, b) \sim\left(103^{\circ} 4^{\prime}\right.$, $-0^{\circ} 42^{\prime}$ ) (cloud B). The emission at $8.3 \mu \mathrm{m}$ correlates very well with the molecular emission. In particular, the brighter emission regions in the MIR coincide with clumps in cloud A [for example at $(l, b) \sim\left(102^{\circ} 43^{\prime},-0^{\circ} 35^{\prime}\right),\left(102^{\circ} 44^{\prime},-0^{\circ} 43^{\prime}\right),\left(102^{\circ} 47^{\prime},-0^{\circ} 44^{\prime}\right)$ and $\left.\left(103^{\circ} 5^{\prime},-0^{\circ} 42^{\prime}\right)\right]$. Cloud $\mathrm{B}$ coincides with the strong radio 


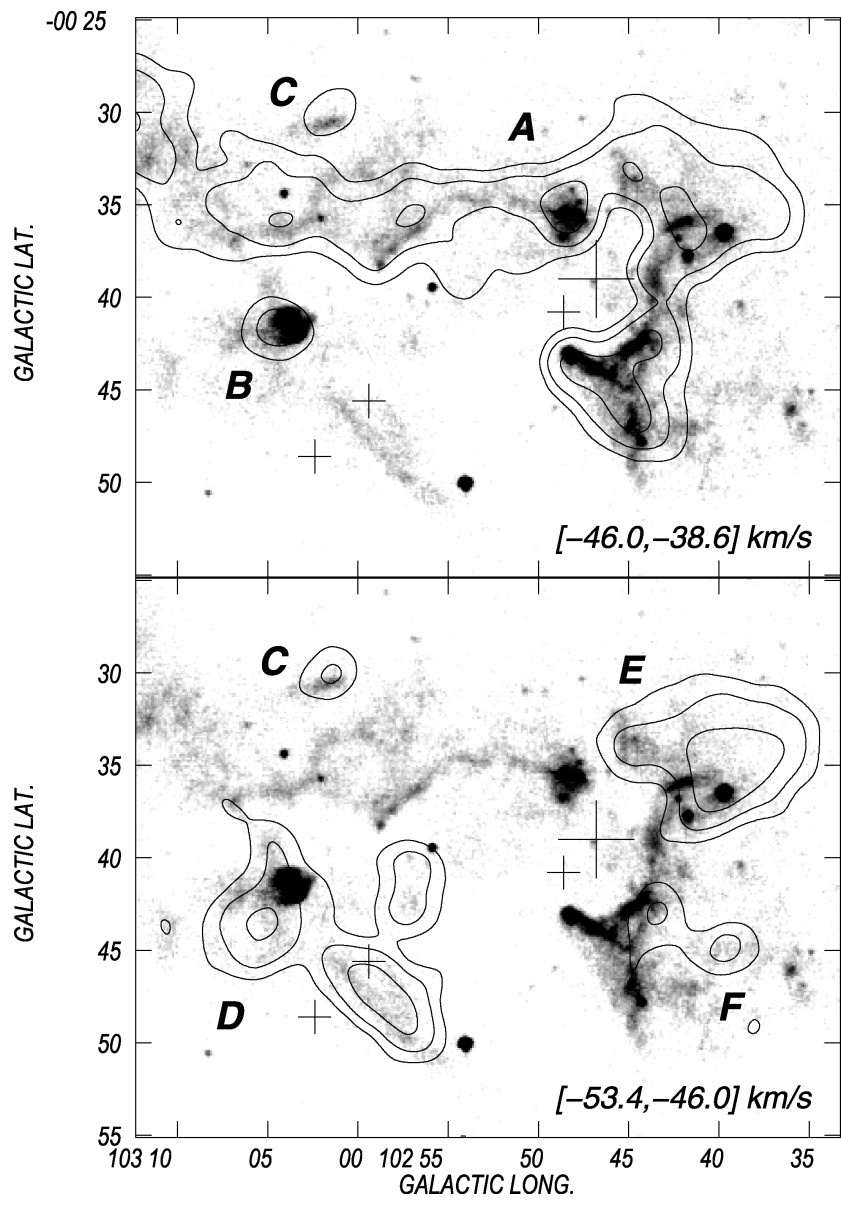

Figure 7. Overlay of the averaged ${ }^{12} \mathrm{CO}$ emission distribution for the molecular clouds between -46.0 and $-38.6 \mathrm{~km} \mathrm{~s}^{-1}$ (upper panel) and between -53.4 and $-46.0 \mathrm{~km} \mathrm{~s}^{-1}$ (lower panel) and the $M S X$ band $A$ emission (greyscale). Contours correspond to 3, 7 and $15 \sigma$. The grey-scale goes from $0.1 \times 10^{-7}$ to $1 \times 10^{-7} \mathrm{~W} \mathrm{~m}^{-2} \mathrm{sr}^{-1}$. The crosses indicate the positions of the massive stars.

source NVSS J222034+561438, which is an YSO candidate. The comparison of the ionized and molecular gas distributions shows that the molecular emission encircles the brightest optical emission regions analysed by Sánchez-Monge et al. (2008).

The lower panel displays the molecular emission between -53.4 and $-46.0 \mathrm{~km} \mathrm{~s}^{-1}$. The $\mathrm{CO}$ emission is clumpy, being concentrated in clouds D, E and F. Cloud $\mathrm{C}$ is also detected in this velocity range. Cloud $\mathrm{E}$ overlaps a section of cloud $\mathrm{A}$ and follows the ringlike feature identified at $8.3 \mu \mathrm{m}$. Cloud $\mathrm{F}$ coincides with part of the IR filament at $(l, b) \sim\left(102^{\circ} 44^{\prime},-0^{\circ} 42^{\prime}\right)$. The section of cloud $\mathrm{D}$ running from $(l, b)=\left(102^{\circ} 56^{\prime},-00^{\circ} 51^{\prime}\right)$ to $(l, b)=\left(103^{\circ} 02^{\prime}\right.$, $\left.-00^{\circ} 44^{\prime}\right)$ coincides with $\mathrm{f} 3$. Cloud $\mathrm{D}$ also displays a clump at $(l, b) \sim$ $\left(103^{\circ} 05^{\prime},-0^{\circ} 44^{\prime}\right)$, which coincides with a region of high optical extinction and is probably connected with cloud B.

Table 5 gives the physical parameters of each molecular cloud. The masses of the different clouds were estimated by integrating the CO line intensity as $W(\mathrm{CO})=\int T_{\mathrm{mb}} \mathrm{d} v=T_{\mathrm{mb}} \Delta v$, where $T_{\mathrm{mb}}$ is the average main beam brightness temperature of the molecular cloud over the velocity interval in which the cloud is observed. To estimate the $\mathrm{H}_{2}$ column density $N\left(\mathrm{H}_{2}\right)$, the relation $X=N\left(\mathrm{H}_{2}\right) / W(\mathrm{CO})=$ $(2.3 \pm 1.2) \times 10^{20} \mathrm{~mol} \mathrm{~cm}^{-2} \mathrm{~K}^{-1} \mathrm{~km}^{-1} \mathrm{~s}$ (Grenier \& Lebrun 1990) was used. The molecular mass was derived from $M\left(\mathrm{M}_{\odot}\right)=4.2 \times$ $10^{20} N\left(\mathrm{H}_{2}\right) \mathrm{d}^{2} \Omega$, where $d$ is the distance in pc and $\Omega$ is the solid angle in steradian. Errors in $T_{\mathrm{mb}}$ and in $N\left(\mathrm{H}_{2}\right)$ arise in background uncertainties, while errors in masses come from background and distance uncertainties. The adopted distance is $3.5 \pm 1.0 \mathrm{kpc}$.

To determine the volume density, we assumed a spherical geometry for all the clouds, except for cloud A, for which an elongated geometry was adopted.

Fig. 8 shows two velocity-position maps of the $\mathrm{CO}$ emission. We have plotted Galactic longitude in the $y$-axis since the molecular morphology is mostly elongated in this direction. The map in the upper panel is the result of integrating within $\Delta b=\left(-0^{\circ} 55^{\prime} 30^{\prime \prime}\right.$, $\left.-0^{\circ} 39^{\prime} 54^{\prime \prime}\right)$, while the map in the bottom panel was integrated within $\Delta b=\left(-0^{\circ} 39^{\prime} 54^{\prime \prime},-0^{\circ} 29^{\prime} 06^{\prime \prime}\right)$. The upper panel shows two clouds at $l \sim 102^{\circ} 45^{\prime}$ and $l \sim 103^{\circ} 00^{\prime}$, having velocities in the range $\Delta v \sim(-40,-45) \mathrm{km} \mathrm{s}^{-1}$ and $(-45,-50) \mathrm{km} \mathrm{s}^{-1}$, respectively (two small cloudlets are also detected at $v \simeq-30 \mathrm{~km} \mathrm{~s}^{-1}$ at $l \sim 102^{\circ} 50^{\prime}$ and $l \sim 103^{\circ} 03^{\prime}$ ). Clouds $\mathrm{F}$ and $\mathrm{D}$ are clearly identified. The bottom panel shows cloud A extending from $-102^{\circ} 35^{\prime}$ to $103^{\circ} 13^{\prime}$. The velocity range goes from $-40 \mathrm{~km} \mathrm{~s}^{-1}$ at $l \sim 103^{\circ} 10^{\prime}$ to $-50 \mathrm{~km} \mathrm{~s}^{-1}$ at $l \sim 102^{\circ} 35^{\prime}$. The image shows some small cloudlets at $l \sim 103^{\circ} 23^{\prime}$ at $v \simeq-38 \mathrm{~km} \mathrm{~s}^{-1}, l<102^{\circ} 40^{\prime}$ with velocities in the range $\sim-8$ to $-5 \mathrm{~km} \mathrm{~s}^{-1}$, and at $l>103^{\circ} 25^{\prime}$ at $v \simeq 0 \mathrm{~km} \mathrm{~s}^{-1}$. Molecular gas with velocities higher than $-10 \mathrm{~km} \mathrm{~s}^{-1}$ is unconnected to Sh2-132.

The agreement in velocity between the ionized and molecular gas and the fact that the molecular emission borders the brightest ionized regions indicate that molecular gas with velocities between -38 and $-54 \mathrm{~km} \mathrm{~s}^{-1}$ is associated with Sh2-132 and the massive stars in the region.

We adopt $-45 \mathrm{~km} \mathrm{~s}^{-1}$ as the systemic velocity of the molecular gas. Taking into account the non-circular motions in this section of the Galaxy shown by Brand \& Blitz (1993), a kinematical distance $d_{\mathrm{k}}=3.0 \pm 1.0 \mathrm{kpc}$ can be estimated, compatible with the distance adopted in Section 1.

\subsection{The distribution of the $\mathrm{HI}$ emission}

The upper panel of Fig. 9 displays the HI emission distribution in the velocity range where the $\mathrm{CO}$ emission is present, i.e. between

Table 5. Physical parameters of the molecular clouds.

\begin{tabular}{lcccccc}
\hline & & & Clouds & & \\
& A & B & C & D & E & F \\
\hline Average $T_{\mathrm{mb}}(\mathrm{K})$ & $1.30 \pm 0.30$ & $0.90 \pm 0.15$ & $0.60 \pm 0.15$ & $1.20 \pm 0.30$ & $1.60 \pm 0.15$ & $0.70 \pm 0.15$ \\
Velocity range $\left(\mathrm{km} \mathrm{s}^{-1}\right)$ & $-38.6,-46.0$ & $-38.6,-46.0$ & $-38.6,-53.4$ & $-46.0,-53.4$ & $-46.0,-53.4$ & $-46.0,-53.4$ \\
$N\left(\mathrm{H}_{2}\right)$ column density $\left(10^{20} \mathrm{~cm}^{-2}\right)$ & $6.50 \pm 1.60$ & $3.30 \pm 0.60$ & $4.20 \pm 1.05$ & $5.90 \pm 2.40$ & $9.0 \pm 0.90$ & $1.95 \pm 0.20$ \\
$\mathrm{H}_{2}$ mass $\left(10^{3} \mathrm{M}_{\odot}\right)$ & $4.25 \pm 1.90$ & $0.80 \pm 0.35$ & $0.04 \pm 0.02$ & $1.30 \pm 0.60$ & $1.55 \pm 0.70$ & $0.95 \pm 0.45$ \\
Volume density $\left(\mathrm{cm}^{-3}\right)$ & $37 \pm 22$ & $2150 \pm 1290$ & $110 \pm 65$ & $95 \pm 55$ & $115 \pm 70$ & $320 \pm 190$ \\
$N\left(\mathrm{H}_{\mathrm{I}}\right)$ column density $\left(10^{21} \mathrm{~cm}^{-2}\right)$ & $3.7 \pm 0.9$ & $3.5 \pm 0.5$ & $3.7 \pm 0.9$ & $3.3 \pm 0.8$ & $3.4 \pm 0.3$ & $3.3 \pm 0.6$ \\
Visual absorption $A_{v}(\mathrm{mag})$ & $2.5 \pm 0.8$ & $2.2 \pm 0.5$ & $2.8 \pm 0.7$ & $2.4 \pm 0.7$ & $2.8 \pm 0.6$ & $2.7 \pm 0.5$ \\
\hline
\end{tabular}




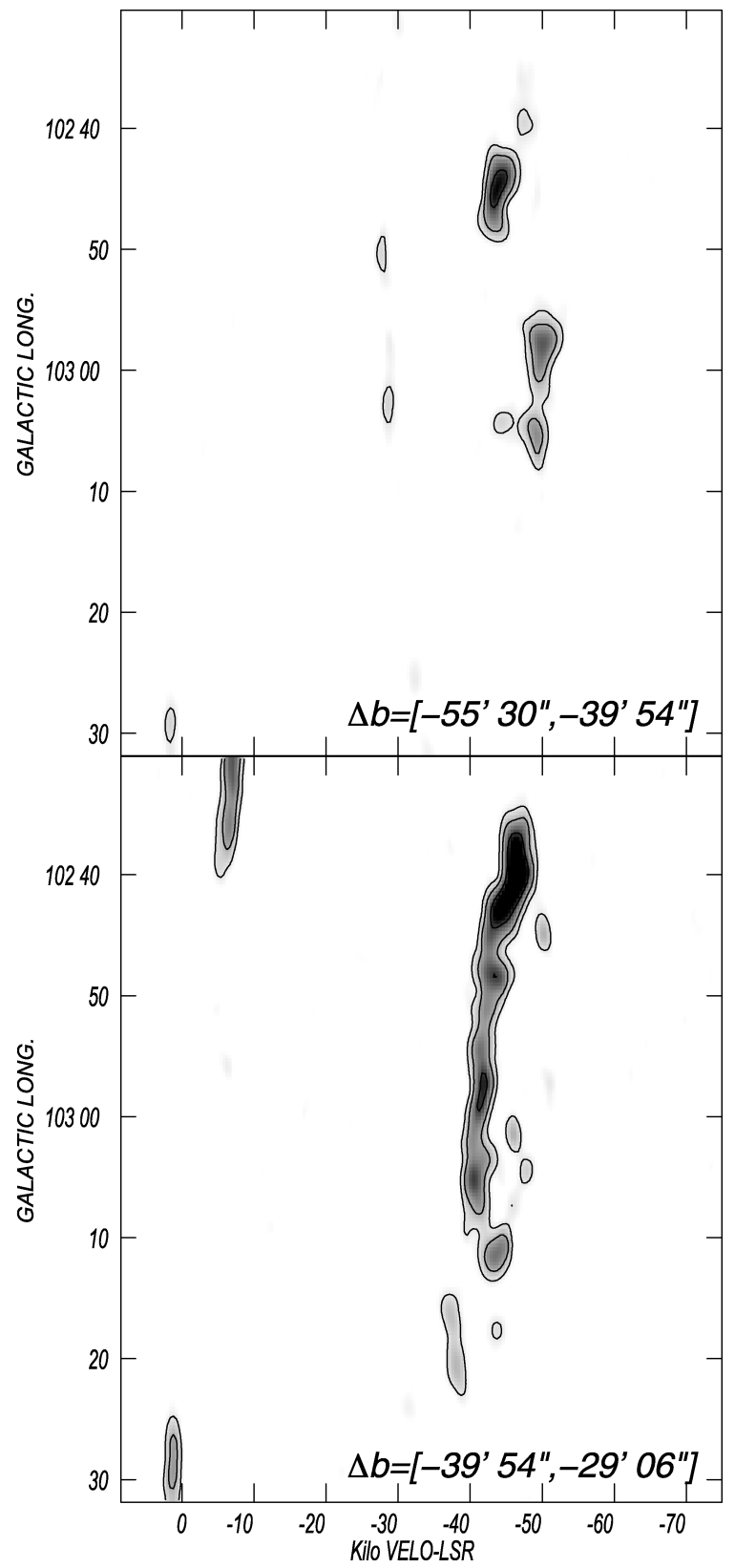

Figure 8. Upper panel: averaged ${ }^{12} \mathrm{CO}$ velocity distribution for $\Delta b=$ $\left(-55^{\prime} 30^{\prime \prime},-39^{\prime} 54^{\prime \prime}\right)$. The grey-scale goes from 0.15 to $3 \mathrm{~K}$. Contours are $0.45,0.75$ and $1.05 \mathrm{~K}$. Bottom panel: averaged ${ }^{12} \mathrm{CO}$ velocity distribution between $\Delta b=\left(-39^{\prime} 54^{\prime \prime},-29^{\prime} 06^{\prime \prime}\right)$. Contours and grey-scale are the same as in the upper panel.

-31.1 and $-58.4 \mathrm{~km} \mathrm{~s}^{-1}$. The white circle has the dimensions of the $\mathrm{H}$ II region. Neither a region lacking $\mathrm{H}_{\mathrm{I}}$ gas coincident in position with Sh2-132 as a whole nor an $\mathrm{H}$ I shell surrounding it is evident in the image, although the small cavity near $(l, b) \simeq\left(102^{\circ} 58^{\prime},-0^{\circ} 40^{\prime}\right)$ is probably linked to shell A.

However, a gradient in the $\mathrm{H}$ I emission distribution is evident for $b<-0^{\circ} 10^{\prime}$. The lower panel displays the emission corresponding to $l=102^{\circ} 50^{\prime}$ from $b=0^{\circ} 00^{\prime}$ to $-1^{\circ} 30^{\prime}$ from the image in the upper panel. $T_{\mathrm{b}}$ and $N(\mathrm{H} \mathrm{I})$ are shown on left- and right-hand $y$-axes, respectively. The image shows that the $\mathrm{H}_{\mathrm{I}}$ brightness temperature is $T_{\mathrm{b}} \simeq 85 \mathrm{~K}$ at $b \simeq-0^{\circ} 15^{\prime}$, while 65 per cent this value is detected
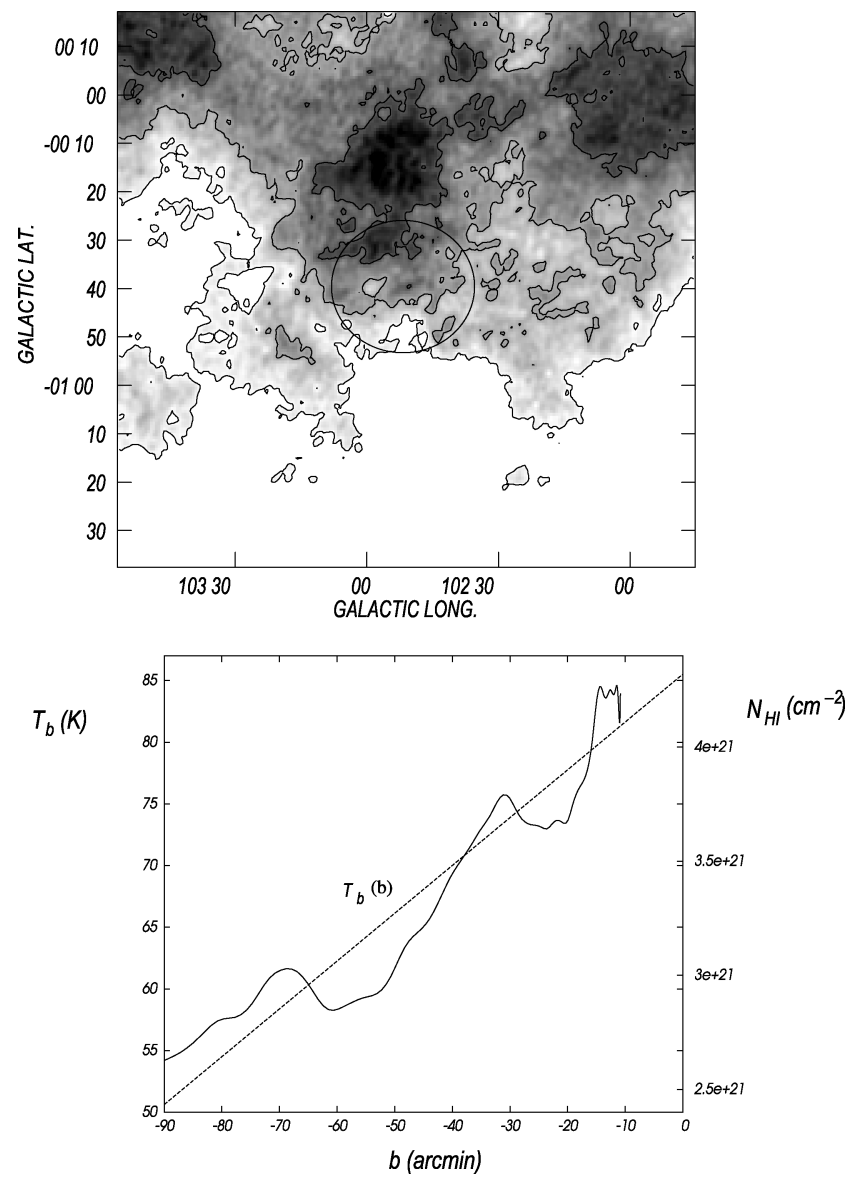

Figure 9. Upper panel: average $\mathrm{H}$ I emission distribution between -31.1 and $-58.4 \mathrm{~km} \mathrm{~s}^{-1}$. The grey-scale goes from 50 to $93 \mathrm{~K}$, while contours correspond to $60,70,80$ and $90 \mathrm{~K}$. The white circle has the dimensions of the $\mathrm{H}_{\text {II }}$ region Sh2-132. Bottom panel: diagram showing $T_{\mathrm{b}}$ versus $b$ from $b=-90^{\prime}$ to $0^{\prime}$ for $l=102^{\circ} 50^{\prime}$ from the image above. The dashed line shows the best fit to the data.

at $b \simeq-1^{\circ} 30^{\prime}$. The fitted curve, showed as a dashed line, confirms the presence of a $T_{\mathrm{b}}$ gradient.

The fitting curve was used to estimate the contribution of the $\mathrm{H}_{\mathrm{I}}$ gas to the visual absorption $A_{v}$. We calculated the visual absorption $A_{v}$ due to the molecular and neutral hydrogen related to the $\mathrm{H}_{\text {II }}$ region by using the relation $\left[N\left(\mathrm{H}_{\mathrm{I}}\right)+2 N\left(\mathrm{H}_{2}\right)\right] / E(B-V)=5.8 \times$ $10^{21}$ atoms $\mathrm{cm}^{-2} \mathrm{mag}^{-1}$ (Bohlin, Savage \& Drake 1978), where $N\left(\mathrm{H}_{\mathrm{I}}\right)$ and $N\left(\mathrm{H}_{2}\right)$ are the $\mathrm{H}_{\mathrm{I}}$ and $\mathrm{H}_{2}$ column densities, respectively, while $E(B-V)$ is the colour excess. We adopted $E(B-V)=$ $A_{v} / 3.05$ and read off $N\left(\mathrm{H}_{\mathrm{I}}\right)(b)$ from the diagram. The estimated $\mathrm{H}_{\mathrm{I}}$ column densities, as well as the derived $A_{v}$ values for each molecular cloud are included in Table 5.

\section{PHOTODISSOCIATED REGIONS}

The presence of PAH emission at the interface between the ionized and molecular material, i.e. at the border of many $\mathrm{H}_{\text {II }}$ regions, suggests that molecular gas is being photodissociated by the UV photons emitted by the massive stars. In the following subsections we analyse the presence of PDRs in two selected areas within the $\mathrm{H}$ II region, one around WR $153 \mathrm{ab}$ and $\mathrm{BD}+55^{\circ} 2722$, linked to shell $\mathrm{B}$, and the other around $\mathrm{LS}+55^{\circ} 39$, linked to shell A. 


\subsection{Region near WR 153ab and $\mathrm{BD}+55^{\circ} 2722$}

The upper panel of Fig. 10 displays an overlay of the DSS-R image and the $\mathrm{CO}$ emission distribution for a small region of Sh2-132 near WR 153ab and BD $+55^{\circ} 2722$. Thin contours correspond to cloud A with velocities in the range -38.6 to $-46.0 \mathrm{~km} \mathrm{~s}^{-1}$, while thick ones delineate clouds $\mathrm{E}$ and $\mathrm{F}$ having velocities between -46.0 and $-53.4 \mathrm{~km} \mathrm{~s}^{-1}$. The crosses mark the position of the stars.

The morphological agreement between the optical emission and the inner borders of the molecular clouds is excellent, particularly for the 4-arcmin optical filament at $(l, b) \sim\left(102^{\circ} 46^{\prime} 30^{\prime \prime},-0^{\circ} 43^{\prime}\right)$, where the contour corresponding to $2.25 \mathrm{~K}$ closely follows the optical emission. Note also that diffuse emission is also encircled by molecular emission. The existence of emission at $8.3 \mu \mathrm{m}$ bordering the ionized region (see Fig. 7) strongly indicates the presence of a PDR.

To confirm the existence of a PDR in this region we compared the distribution of the ionized and molecular gas, and that of PAHs. After convolving the DSS-R and $M S X$ band $A$ images to the CO beam (46 arcsec), we averaged the optical, molecular and band $A$ emis-
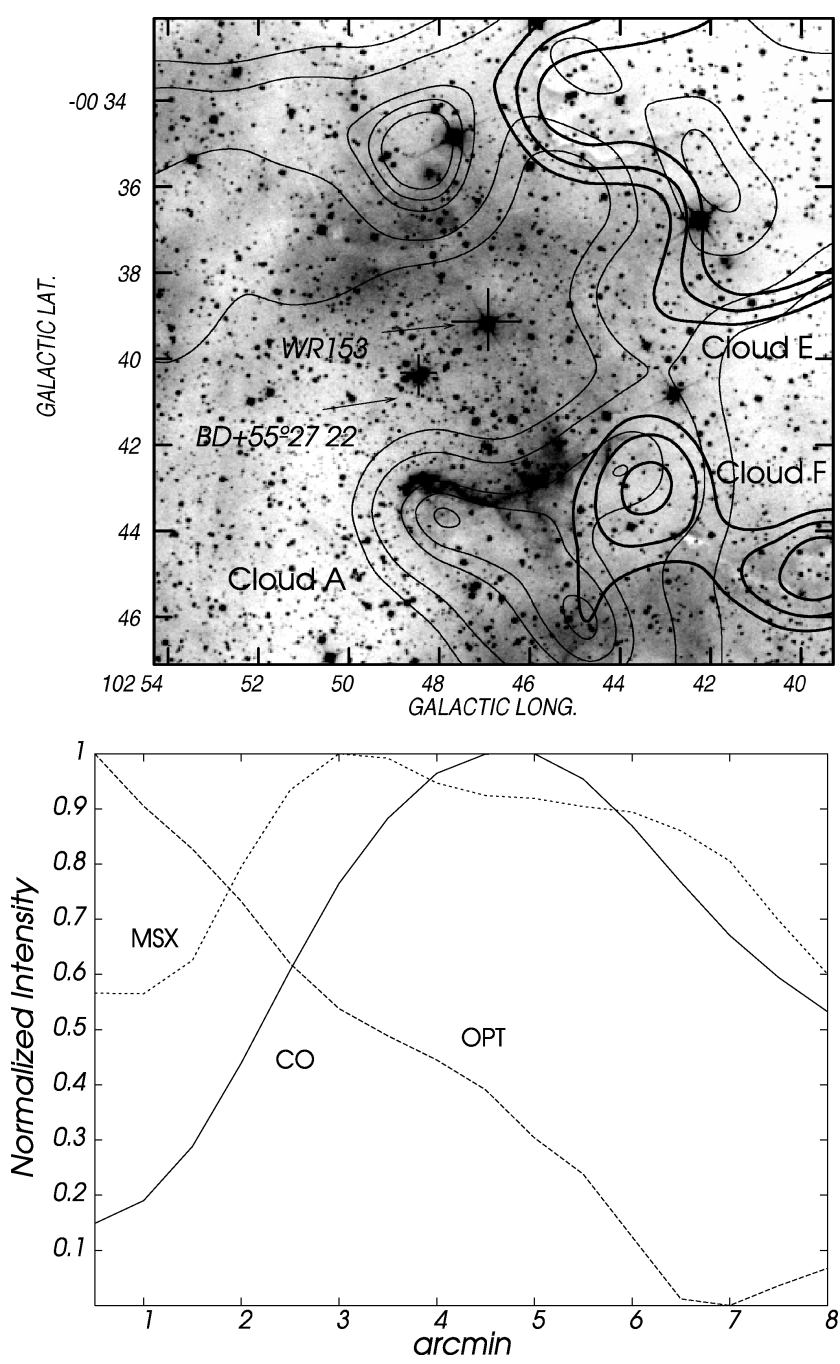

Figure 10. Upper panel: overlay of the DSS-R image and the CO emission in the region near WR 153 and $\mathrm{LS}+55^{\circ} 2722$, indicated as crosses. Thin lines delineate cloud A and thick lines, clouds $\mathrm{E}$ and F. Bottom panel: normalized intensities of the optical, MSX band $A$ and $\mathrm{CO}$ emissions in concentric rings as a function of the distance to the WR star. sions in concentric rings from the position of the WR star within an angle of $300^{\circ}$ from $(l, b) \sim\left(102^{\circ} 52^{\prime},-0^{\circ} 38^{\prime}\right)$ clockwise up to $(l, b) \sim\left(102^{\circ} 49^{\prime},-0^{\circ} 33^{\prime}\right)$. The result of this average is displayed in the bottom panel of the figure, where intensities in the different bands are normalized to the maximum value of each band. The values in the abscissae indicate distance from the WR star. The diagram reveals a clear stratification, with the optical emission peaking near the stellar position, and the emission at $8.3 \mu \mathrm{m}$ peaking $3 \mathrm{arcmin}$ far from the star. The molecular emission increases up to 6 arcmin from the star, where the maximum is present. The observed distribution is typical of molecular clouds exposed to UV radiation, which photodissociates them (van der Werf et al. 1996).

\subsection{Region near $\mathrm{LS}+55^{\circ} 39$}

The region around $\mathrm{LS}+55^{\circ} 39$ is particularly interesting. The upper panel of Fig. 11 shows that the star is close to a bright optical filament of $\sim 2$ arcmin in size. The fact that the optical filament partially follows the border of cloud A indicates that the UV photons of the star are photodissociating and ionizing the dense gas. The
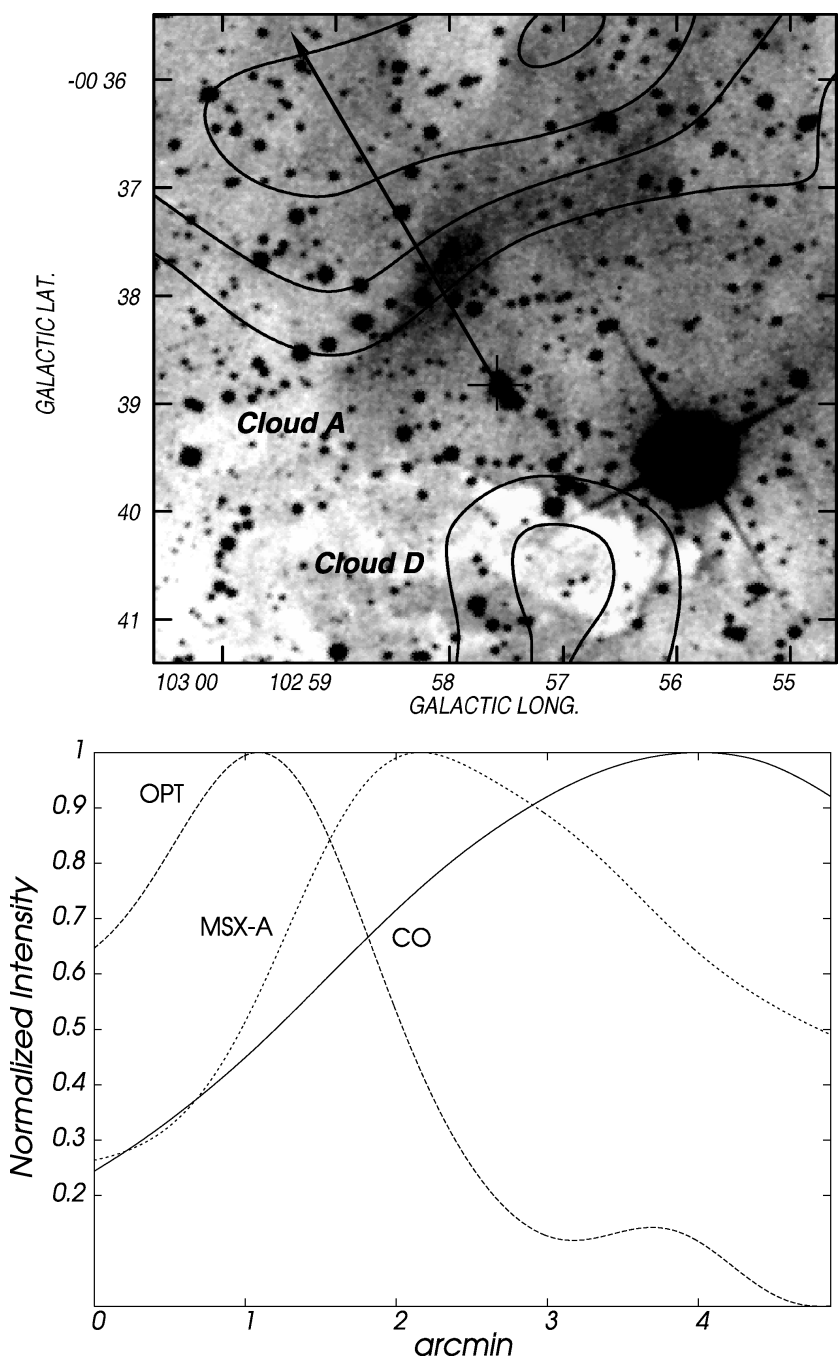

Figure 11. Upper panel: overlay of the DSS-R image and the CO emission in the region near $\mathrm{LS}+55^{\circ} 39$, indicated by a cross. Thin lines delineate clouds A and D. Bottom panel: normalized intensities of the optical, MSX band $A$ and $\mathrm{CO}$ emissions in concentric rings as a function of the distance to the $\mathrm{B}$ star. 
high extinction region at $(l, b) \sim\left(102^{\circ} 57^{\prime},-0^{\circ} 40^{\prime}\right)$ coincides with a section of cloud D.

With the aim of investigating the presence of a PDR near the optical filament, we plotted the normalized intensity of optical, $M S X$ band $A$, and CO emissions as a function of the distance to $\mathrm{LS}+55^{\circ} 39$. The plotted emission corresponds to the region indicated by the black arrow in the upper panel. As for the region around WR $153 \mathrm{ab}$ and $\mathrm{BD}+55^{\circ} 2722$, the optical emission peaks closer to the excitation source than the emission in $M S X$ band $A$ and the molecular emission, revealing the presence of a PDR.

Taking into account that $\mathrm{LS}+55^{\circ} 39$ is a B0V star with $\log Q\left(H^{0}\right)=46.23$ (Vacca et al. 1996), and assuming that it is immersed in an ionized ISM with a mean electron density $n_{\mathrm{e}}=$ $20 \mathrm{~cm}^{-3}$, we can estimate the Strömgren radius $r_{\mathrm{s}}$ of the $\mathrm{H}_{\mathrm{II}}$ region created by the star from $3 Q\left(H^{0}\right) /\left[4 \pi \alpha_{B} n_{\mathrm{e}}^{2}\right]=r_{\mathrm{s}}^{3}$ (Mezger \& Henderson 1967), where $\alpha_{B}=2 \times 10^{-13} \mathrm{~cm}^{-3} \mathrm{~s}^{-1}$. The result is $r_{\mathrm{s}}=5.3 \mathrm{pc}$. Bearing in mind that the angular distance from the star to the optical filament is $2.2 \mathrm{arcmin}$, or $2.25 \pm 0.7 \mathrm{pc}$ at $3.5 \pm 1.0 \mathrm{kpc}$, we can conclude that the B-type star is responsible for ionizing the gas in the region and contributing to the photodissociation of the molecular gas.

\section{SCENARIO AND CONCLUSIONS}

In this paper we have analysed the distribution of the ionized, neutral atomic and molecular gas and that of the interstellar dust in Sh2-132.

This paper revealed the presence of a number of molecular clouds encircling the ionized nebula, with a mass of $9000 \mathrm{M}_{\odot}$. The velocity of the molecular gas, which is in the range from -38.6 to $-53.4 \mathrm{~km} \mathrm{~s}^{-1}$ coincides with the velocities of the $\mathrm{H} \alpha$ line, and $\mathrm{He}$ and $\mathrm{H}$ radio recombination lines, strongly indicating that the ionized gas is associated with the nebula and interacting with the ionized gas.

The ring-like appearance of the gas and dust distribution in the environs of the WR star suggests the action of stellar winds that sweep up and compress the gas. The mechanical energy $E_{\mathrm{w}}$ released by the multiple system WR 153ab into the ISM can be roughly estimated taking into account the two WR components of the binary system during the duration of the WR phase of a massive star, the previous main-sequence phase of the current WR stars, and the two O-star components. We adopt a mass-loss rate $\log (\dot{M})=-6.64$ and a terminal velocity $v_{\infty}=1950 \mathrm{~km} \mathrm{~s}^{-1}$, corresponding to a $08.5 \mathrm{~V}$ star (Smith et al. 2002) as parameters of the stellar wind of the O-type components. For the WR components we adopt a conservative mass-loss rate $\dot{M}=5 \times 10^{-6} \mathrm{M}_{\odot} \mathrm{yr}^{-1}$ and a terminal velocity $v_{\infty}=2000 \mathrm{~km} \mathrm{~s}^{-1}$. Adopting lifetimes of $3 \times 10^{6}$ and $0.5 \times 10^{6} \mathrm{yr}$ for the $\mathrm{O}$ and WR phases of the stars, respectively (Conti \& Vacca 1990; Meynet \& Maeder 2005), $E_{\mathrm{w}}$ results to be $\sim 7.2 \times 10^{50} \mathrm{erg}$.

Considering the molecular clouds E, F and a section of cloud A, and the dust and ionized components, with an expansion velocity of $7.4 \mathrm{~km} \mathrm{~s}^{-1}$, the kinetic energy of shell B is $\sim 2.80 \times 10^{48} \mathrm{erg}$. This result indicates that the multiple system can shape shell $\mathrm{B}$.

The emission at $8.3 \mu \mathrm{m}$ appears concentrated in a ring-like structure bordering the brightest sections of the nebula, indicating that molecular material is being photodissociated at the interface between the ionized and molecular gas. This configuration resembles the IR bubbles analysed by Watson et al. (2008). To confirm the presence of PDRs we have analysed in some detail two regions within the complex. One in Shell B around WR 153ab and BD+55 2722, and the other in Shell A around $\mathrm{LS}+55^{\circ} 39$. The relative positions of the ionized gas, PAHs and molecular gas in these regions show the stratification typical in PDRs. Thus, the distribution of $\mathrm{H}_{2}$ plays a main role in shaping the $\mathrm{H}$ II region.

The emission distribution at $60 \mu \mathrm{m}$ shows the same ring-like structure, along with emission inside the ring. The presence of emission in the FIR inside the $\mathrm{H}_{\text {II }}$ region, which originates from large dust grains, is compatible with the existence of PDRs since PAHs are destroyed inside $\mathrm{H}_{\text {II }}$ regions, while large grains are not.

The number of UV photons emitted by the massive stars in the region is seven times higher than the UV photons used to ionize the gas, indicating that the massive stars are responsible for the ionization of the region. Clearly, a large fraction of the available UV photons are used in the heating of the interstellar gas and in the photodissociation of the dense material.

Different studies of $\mathrm{H}_{\text {II }}$ regions such as those towards RCW 79 (Zavagno et al. 2006), RCW 82 (Pomarés et al. 2009) and Sh2-212 (Deharveng et al. 2008) show how the interaction between the massive stars with the surrounding molecular material induces star forming regions through the 'collect and collapse' process (Elmegreen \& Lada 1977) in the borders of these H II regions. For example, the paper on RCW 79 (Zavagno et al. 2006) reveals the formation of YSOs inside pre-existing molecular cores located at the border of the ionized region. By using the analytical model by Whitworth et al. (1994) to determine the time at which the fragmentation occurs and the size of the structure at that time, they conclude that the collect and collapse process may not be the only one at work. Sh2-212 (Deharveng et al. 2008) and Sh2-104 (Deharveng et al. 2003) represent good examples of stellar formation in which the molecular clouds surround the $\mathrm{H}_{\text {II }}$ regions and the PAH emission is located at the interface between the ionized and the molecular gases. In particular, Sh2-104 is encircled by a molecular ring showing four dense molecular clouds containing many dense cores. This configuration is a strong evidence in favour of the triggering star formation process described by the 'collect and collapse' model. In all of the preceding examples, we have an $\mathrm{H}$ II region generated by an O-type star or a cluster of massive stars.

The region around Shell B presents a similar interstellar scenario as the preceding examples, i.e. an optical shell bounded by a strong arc-like feature of PAH emission which are surrounding by molecular material (clouds A, E and F). In such a scenario, star formation can be induced by the action of the massive stars through the ionizing flux and stellar winds. Indeed, the presence of YSOs towards the molecular clouds linked to Sh2-132 indicates that star formation is very active in this region of the Galaxy (Vasquez et al., in preparation).

\section{ACKNOWLEDGMENTS}

We acknowledge the anonymous referee of her/his comments. This project was partially financed by the Consejo Nacional de Investigaciones Científicas y Técnicas (CONICET) of Argentina under projects PIP 112-200801-02488 and PIP 112-200801-01299, Universidad Nacional de La Plata (UNLP) under project 11/G093, Universidad de Buenos Aires under project UBACyT X482 and Agencia Nacional de Promoción Científica y Tecnológica (ANPCYT) under projects PICT 00812 and 2007-00902. The DSS was produced at the Space Telescope Science Institute under US Government grant NAGW-2166. This work was partly (SP) supported by the Natural Sciences and Engineering Research Council of Canada (NSERC) and the Fonds FQRNT of Québec. The DRAO Synthesis Telescope is operated as a national facility by the National Research Council of Canada. The CGPS is a Canadian project with international partners and is supported by grants from NSERC. Data 
from the CGPS are publicly available through the facilities of the Canadian Astronomy Data Centre (http://cadc.hia.nrc.ca) operated by the Herzberg Institute of Astrophysics, NRC.

\section{REFERENCES}

Bakes E. L. O., Tielens A. G. G. M., 1998, ApJ, 499, 258

Bohlin R. C., Savage B. D., Drake J. F., 1978, ApJ, 224, 132

Brand J., Blitz L., 1993, A\&A, 275, 67

Cappa C. E., Vasquez J., Pineault S., Cichowolski S., 2010, MNRAS, 403, 387

Chaisson E. J., 1976, in Avrett E. H., ed., Frontiers of Astrophysics. Harvard Univ. Press, Cambridge, p. 259

Chu Y.-H., 1981, ApJ, 249, 195

Chu Y.-H., Treffers R. R., 1981, ApJ, 250, 615

Chu Y.-H., Treffers R. R., Kwitter K. B., 1983, ApJS, 53, 937

Churchwell E., Walmsley C. M., 1973, A\&A, 23, 117

Condon J. J., Cotton W. D., Greisen E. W., Yin Q. F., Perley R. A., Taylor G. B., Broderick J. J., 1998, AJ, 115, 1693

Conti P. S., Vacca W. D., 1990, AJ, 100, 431

Deharveng L., Lefloch B., Zavagno A., Caplan J., Whitworth A. P., Nadeau D., Martín S., 2003, A\&A, 408, 25

Deharveng L., Lefloch B., Kurtz S., Nadeau D., Pomarés M., Caplan J., Zavagno A., 2008, A\&A, 482, 585

Douglas J. N., Bash F. N., Bozyan F. A., Torrence G. W., Wolfe C., 1996, AJ, 111, 1945

Elmegreen B. G., Lada C. J., 1977, ApJ, 214, 725

Esteban C., Rosado M., 1995, A\&A, 304, 491

Fich M., Blitz M., Stark A. A., 1989, ApJ, 342, 272

Fich M., Dahl G. P., Treffers R. R., 1990, AJ, 99, 622

Foster T., Routledge D., 2003, ApJ, 598, 1005

Georgelin Y. M., Georgelin Y. P., 1976, A\&A, 49, 57

Gower J. F. R., Scott P. F., Wills D., 1967, MNRAS, 71, 49

Grenier I. A., Lebrun F., 1990, ApJ, 360, 129

Habing H. J., 1968, Bull. Astron. Inst. Astrophys., 204, 253

Harten R. H., Felli M., Tofani G., 1978, A\&A, 70, 205

Heckathorn J. N., Bruhweiler F. C., Gull T. R., 1982, ApJ, 252, 230

Hogerheijde M. R., Jansen D. J., van Dishoeck E. F., 1995, A\&A, 294, 792

Junkes N., Fuerst E., Reich W., 1992, A\&A, 261, 289

Kaufman M. J., Wolfire M. g. Hollenbach D. J., Luhman M. L., 1999, ApJ, 527,795

Landolt-Börmstein, 1982, in Hellwege K. H., ed., Astronomy and Astrophysics, Vol. 2, Astronomy, Astrophysics and Space Research, Group VI, Numerical Data and Functional Relationships in Science and Technology

Lumsden S. L., Hoare M. G., Oudmaijer R. D., Richards D., 2002, MNRAS, 336,621
Massey P., 1981, PASP, 93, 549

Meynet G., Maeder A., 2005, A\&A, 429, 581

Mezger P. G., Henderson A. P., 1967, ApJ, 147, 471

Miller G. J., Chu Y.-H., 1993, ApJS, 85, 137

Panov K. P., Seggewiss W., 1990, A\&A, 227, 117

Pomarés M. et al., 2009, A\&A, 494, 987

Povich M. S. et al., 2007, ApJ, 660, 346

Price S. D., Egan M. P., Carey S. J., Mizuno D. R., Kuchar T. A., 2001, AJ, 121,2819

Quireza C., Rood R. T., Balser D. S., Bania T. M., 2006a, ApJS, 165, 338

Quireza C., Rood R. T., Bania T. M., Balser D. S., Maciel W. J., 2006b, ApJ, 653,1226

Reynolds R. J., 1988, ApJ, 333, 341

Sánchez-Monge A., Palau A., Estalella R., Beltrán M. T., Girart J. M., 2008, A\&A, 485, 497

Schwartz P. R., 1982, ApJ, 252, 589

Scoville N. Z., Kwan J., 1976, ApJ, 206, 718

Sharpless S., 1959, ApJS, 4, 257

Smith L. F., Shara M. M., Moffat A. F. J., 1998, MNRAS, 281, 163

Smith L. J., Norris R. P. F., Crowther P. A., 2002, MNRAS, 337, 1309

Sota A., Maíz-Apellániz J., Walborn N. R., Shida R. Y., 2008, Revista Mexicana Astron. Astrofisica, 33, 56

Sternberg A., Dalgarno A., 1989, ApJ, 338, 197

Sternberg A., Dalgarno A., 1995, ApJS, 99, 565

Taylor A. R. et al., 2003, AJ, 125, 3145

Tielens A. G. G. M., Hollenbach D., 1985, ApJ, 291, 722

Vacca W. D., Garmany C. D., Shull J. M., 1996, ApJ, 460, 914

van der Hucht K. A., 2001, New Astron. Rev., 45, 135

van der Werf P. P., Stutzki J., Sternberg A., Krabbe A., 1996, A\&A, 313, 633

Waldram E. M., Yates J. A., Riley J. M., Warner P. J., 1996, MNRAS, 282, 779

Watson C., Povich M. S., Churchwell E. B., Babler B. L., Chunev G. et al., 2008, ApJ, 681, 1341

Wegner W., 1994, MNRAS, 270, 229

Whittet D. C. B., Scarrot S. M., 1994, Dust in the Galactic Environment. Cambridge Univ. Press, Cambridge

Whitworth A. P., Bhattal A. S., Chapman S. J., Disney M. J., Turner J. A., 1994, A\&A, 290, 421

Zavagno A., Deharveng L., Comerón F., Brand J., Massi F., Caplan J., Russeil D., 2006, A\&A, 446, 171

This paper has been typeset from a $\mathrm{T}_{\mathrm{E}} \mathrm{X} / \mathrm{L} \mathrm{T}_{\mathrm{E}} \mathrm{X}$ file prepared by the author. 